\title{
Effet d'écran d'une thermocline Observations faites sur la bouée-laboratoire
}

\author{
par J,-A. Gonella \\ Laboratoire d'Océanographie Physique du Muséum \\ 43, rue Cuvier, 75231 Paris Cedex 05
}

\section{I - Introduction Hypothèse de base}

Les observations du courant, recueillies à bord de la bouée-laboratoire mouillée en Méditerranée occidentale nord de 1964 à 1970 , soit au point $\mathrm{A}\left(42^{\circ} 47^{\prime} \mathrm{N}\right.$ et $\left.07^{\circ} 29^{\prime} \mathrm{E}\right)$ ou au point $\mathrm{B}\left(42^{\circ} 14^{\prime} \mathrm{N}\right.$ et $\left.05^{\circ} 35^{\prime} \mathrm{E}\right)$, montrent que les mouvements des couches superficielles ne présentent pas les mêmes caractéristiques en été et en hiver, tant dans les hodographes intégrés (fig. $1 \mathrm{a}, \mathrm{b}$ ) que dans les spectres (fig. 2 a, b).

Cette différence doit être associée à l'évolution de la stratification de l'hiver à l'été. Dans cette zone exceptionnelle, se rencontre, en hiver, un milieu pratiquement homogène de la surface jusqu'au fond, la variation relative de densité est de l'ordre de $10^{-4}$ à $10^{-6}$. Par contre, en été, la stratification est très nette et la variation relative de densité entre la surface et le bas de la thermocline est de l'ordre de $10^{-3}$.

En régime d'hiver, c'est-à-dire en milieu homogène, les mesures ne montrent aucune corrélation entre vents et courants (fig. 1 b); par contre, au même moment, il a été observé dans cette zone, vers $800-1000 \mathrm{~m}$, des vitesses verticales atteignant un ordre de grandeur comparable à celui des vitesses horizontales (Voorhis, Gascard). Ceci s'expliciue par le fait que, dans un océan très faiblement stratifie $\left(\Delta \rho / \rho \sim 10^{-6}\right)$, la composante verticale de la force de Coriolis est du même ordre de grandeur que la poussée d'Archimède s'exerçant sur une particule fluide de cet océan. En d'autres termes, ceci signifie que la fréquence de Coriolis $f(f=2 \Omega \sin \varphi ; \Omega=$ vitesse de rotation de la Terre, $\varphi$ la latitude du lieu) est du même ordire de grandeur que la fréquence de Brunt-Väisälä $N$, qui est reliée à la stratification de l'océan $\left[N^{2}=-(g / \rho)(\partial \rho / \partial z) ; g\right.$ pesanteur, $\rho$ masse volumique, $z$ altitude].
Par contre, en présence d'une stratification prononcée dans les couches superficielles, par exemple couche de mélange d'une vingtaine de mètres d'épaisseur au-dessus d'une forte thermocline (gradient thermique de l'ordre du degré par mètre), la relation vent-courant semble se rapprocher beaucoup plus des résultats de la théorie d'Ekman. En effet, en régime d'été, nous avons remarqué :

- la présence quasi-permanente des oscillations d'inertie dans la couche de mélange (ex.: fig. 1 a; fig. 3 en haut, courant à $5 \mathrm{~m}$ );

- au cours d'un coup de vent (fig. 3), un courant moyen de dérive du même ordre de grandeur dans toute la couche de mélange et une déviation de, ce courant par rapport au verì supérieure à $45^{\circ}$;

- et enfin, le manque de cohérence entre les courants de part et d'autre de la thermocline (fig. 3 en haut, courant à $5 \mathrm{~m}$; en bas, courant à $60 \mathrm{~m}$; thermocline aux environs de $25 \mathrm{~m}$ ).

Ces différentes remarques nous ont amenés à penser que la thermocline constitue un écran au transfert de la quantité de mouvement. Nous allons examiner maintenant ce que donne la théorie d'Ekman en régime impulsionnel pour un océan présentant une couche de mélange glissant sans frottement sur la couche sous-jacenie.

\section{II - Equation du mouvement Solution générale. Interprétation}

L'étude locale du courant s'établissant loin des côtes sous l'action du vent, peut être abordée avec la plupart des hypothèses d'Ekman (1902) : océan horizontaloment illimité, mouvement plan, gradient horizontal de pression nul, coeffi- 


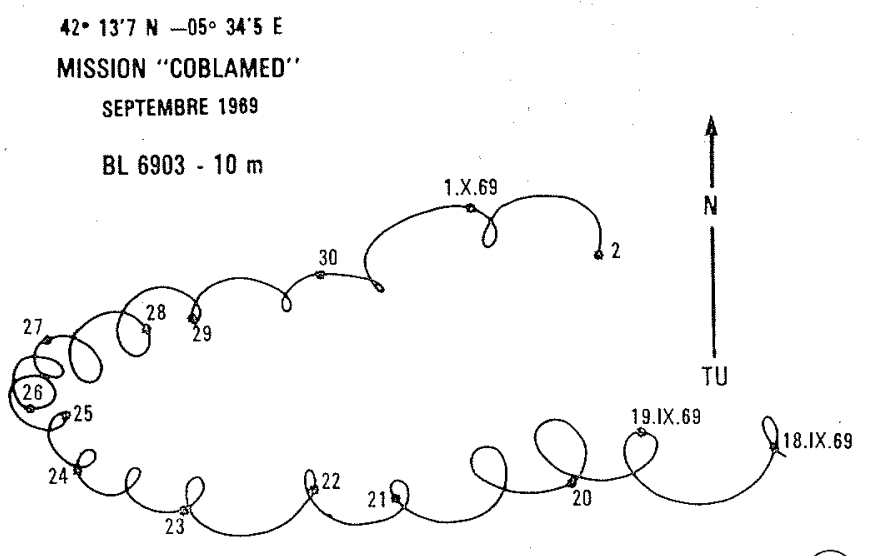

a)

\section{官 1/ Hodographes intégrés des \\ courants de surface}

(a) régime d'ééé;

(b) régime d'hive

2/ Spectres des courants de surface :

(a) régime d'été;

(b) régime d'hiver.


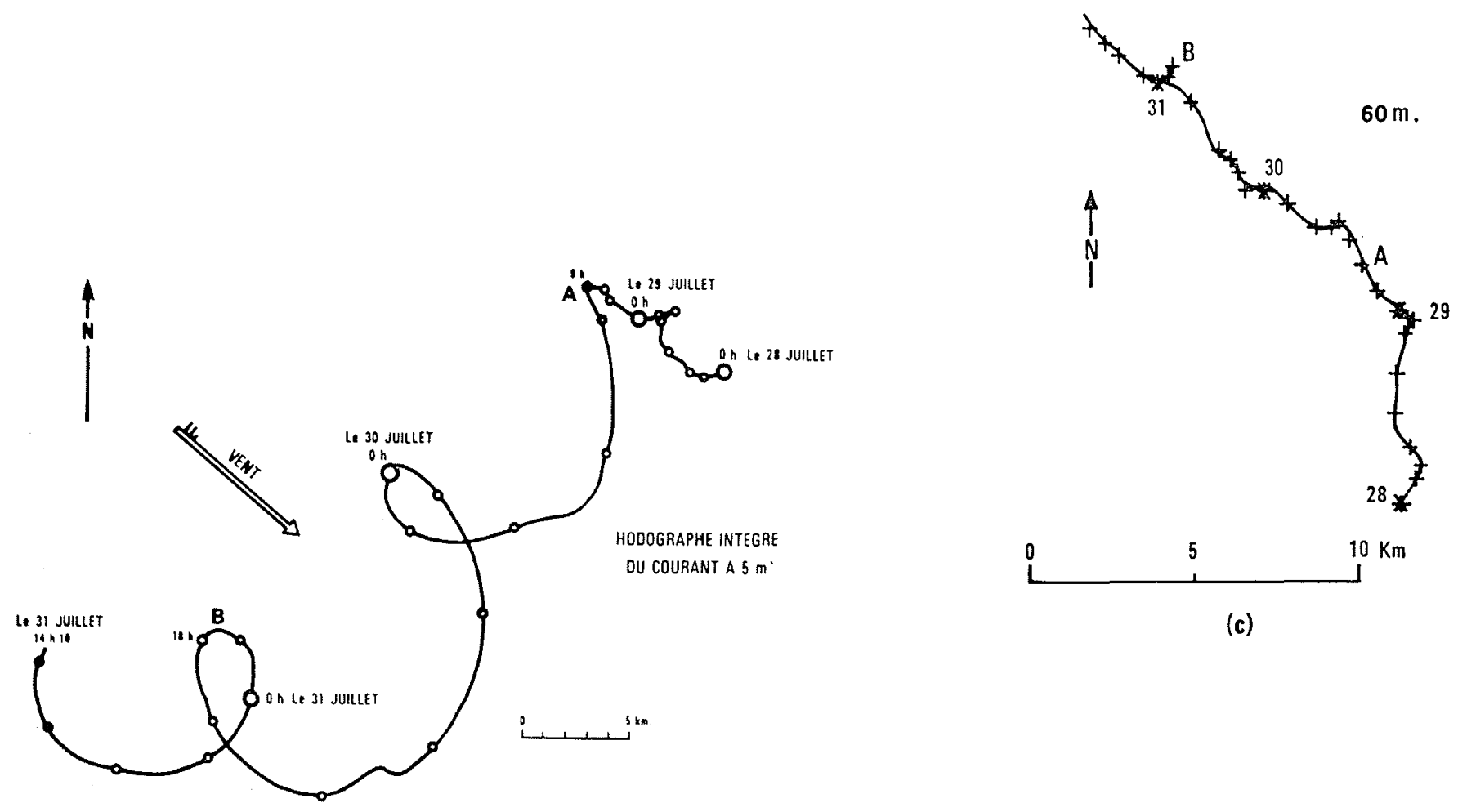

(a)

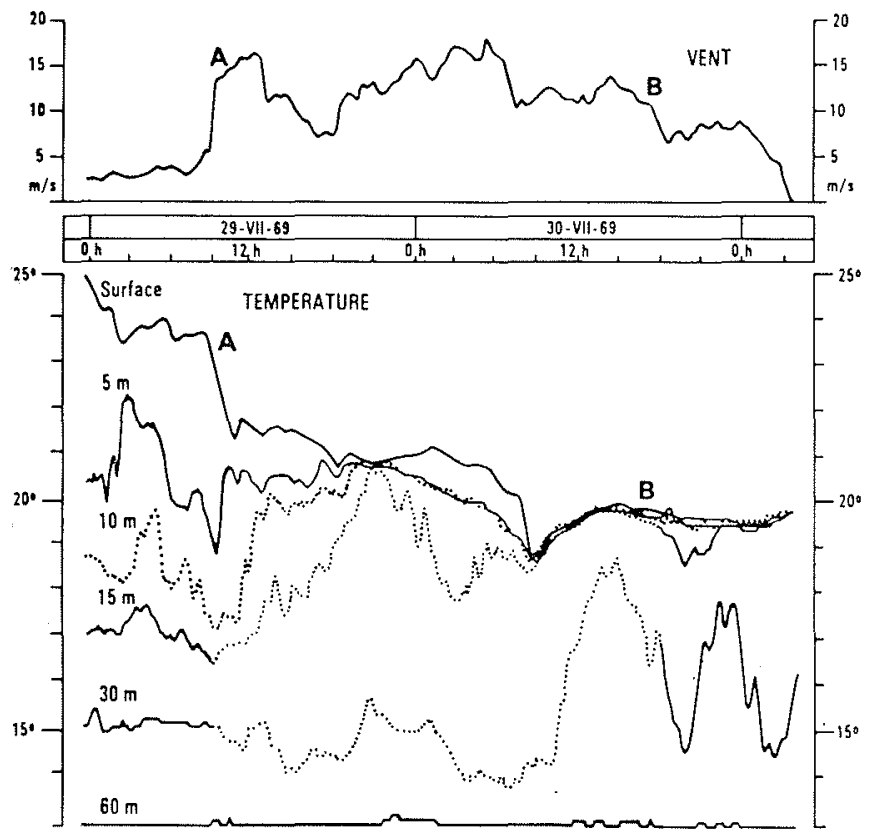

(b)
3/ On a représenté ici l'influence d'un coup de vent (le "Mistral") sur le courant marin près de la surface et sur l'évolution de la température à différentes profondeurs.

(a) La réponse dynamique: on a reconstitué l'hodographe intégré, c'est-à-dire la trajectoire qu'aurait décrite un flotteur s'il était animé à chaque instant d'une vitesse égale à celle du courant mesuré à $5 \mathrm{~m}$ d'immersion. Le mouvement est constitué d'abord par un mouvement d'ensemble vers le SW à droite de la direction du vent qui venait du NW. Cette déviation du courant a droite du vent et les «boucles» dans la trajectoire sont dues à la rotation terrestre qui " polarise " les mouvements océaniques; les "boucles" sont décrites dans le sens des aiguilles d'une montre pour l'hémisphère Nord, dans le sens contraire pour l'hémisphère Sud.

(b) La réponse thermique : les courbes représentent l'évolution de la température de la mer à différentes immersions-surface, $5,10,15,30$ et $60 \mathrm{~m}$. La partie $A B$ correspond à la durée du coup de vent. On note l'effet du mélange provoqué par les vagues engendrées par le coup de vent : la couche homotherme jusqu'à $15 \mathrm{~m}$ au bout d'une dizaine d'heures. A $60 \mathrm{~m}$, le coup de vent n'a pas d'effet sensible car la thermocline - zone de transition qui sépare en été les eaux de surface plus chaudes des eaux profondes - a joué le rôle d'écran et a empêché le transfert de toute perturbation. Cet exemple montre clairement que courants et températures au voisinage de la surface des océans peuvent changer considérablement en quelques heures. La conduite de certaines opérations à la mer (implantation de plate-forme ou de pipe-line, pêche et détection sous-marine) nécessite la connaissance préalable de ces phénomènes dynamiques et thermiques.

(c) Sous la thermocline, à $60 \mathrm{~m}$ d'immersion, on ne voit pas de relation nette et claire entre le vent et le courant à cette immersion. En effet, l'hodographe intégré du courant à $60 \mathrm{~m}$ ne présente aucune modification en liaison avec le début du coup de vent. 
cient de viscosité cinématique turbulente $v$ et paramètre de Coriolis $f$ constants.

Avec les hypothèses faites ci-dessus, le mouvement ne dépend pluis que de l'immersion $z$ et du temps $t$ et son équation au sens des distributions et dans l'hémisphère Nord s'écrit :

$$
\frac{\partial u}{\partial t}+i f u-v \frac{\partial^{2} u}{\partial z^{2}}=q(z, t)+u(z, 0) \delta(t)
$$

où $u=u_{1}+i u_{2}$ est le complexe associé au vecteur courant; $q(z, t)$ la distribution des accélérations dues aux forces extérieures (ex.: tension du vent);

$\delta(t)$ la distribution de Dirac;

et $u(z, 0) \delta(t)$ la distribution initiale des vitesses dans la couche de mélange.

La disiribution des accéiérations $q(z, t)$ dépend de la tension $\tau$ du vent en surface et des conditions au fond de la couche de mélange. Dans le cas d'une conche superficielle de profondeur $h$ glissant sans frottement sur la couche sous-jacente (tension nulle à la profoncieur $z=-h$ ), le mouvement n'aura lieu que dans le domaine de profondeur $z$ comprise entre 0 et $-h$. L'équation du mouvement, au terme ifu près, est la même que celle de la chaleur; la solution élémentaire est donc, au facteur $e^{-i f t}$ près, la solution élémentaire de l'équation de la chaleur, soit:

$$
G(z, t)=Y(t) e^{--i j t} \frac{e^{-z^{2} / 4 n t}}{\sqrt{4 \pi \nu t}}
$$

par suite, la solution générale est :

$$
u(z, t)=G(z, t) \div[q(z, t)+u(z, 0) \delta(t)]
$$

Nous allons résumer les résultats théoriques obtenus (Gonella). Le tableau I donne pour tout $\omega \neq-f$ l'expression mathématique de la distribution des accélérations, de la solution élémentaire, de la transformée de Fourier de la réponse et, enfin, de la phase $\phi_{\omega}$ entre l'excitation (tension du vent) et la réponse du courant dans les trois cas suivants: transport de masse, océan de profondeur infinie (généralisation du problème d'Ekman à toutes les vitesses angulaires $\omega)$ et océan ayant une couche de mélange d'épaisseur $h$ glissant sans frottement sur la couche sous-jacente. Le tableau fait ressortir la discontinuité de la phase $\phi_{\omega}$ à la vitesse angulaire $-f$, vitesse angulaire propre du système qui explique l'apparition d'oscillations d'inertie quelle que soit la fréquence de l'excitation.

Dans la figure 4 , nous avons représenté pour $f+\omega>0$, l'allure de $u_{\omega}$ à une profondeur déterminée $(z=0$ ou $z=-h$ ), lorsque $h$ varie de zéro à l'infini. L'unité de

\begin{tabular}{|c|c|c|c|}
\hline \multicolumn{4}{|c|}{ EXTENSION DE LA THEORIE D'EKMAN } \\
\hline & $\begin{array}{l}\text { Transport } \\
\text { de masse }\end{array}$ & Profondeur $h$ infinie & Profondeur finie $h$ \\
\hline $\begin{array}{c}\text { Distribution } \\
\text { des accélérations }\end{array}$ & $Y(t) \frac{\tau(t)}{\rho}$ & $2 Y(t) \frac{\tau(t)}{\rho} \delta(z)$ & $2 Y(t) \frac{\tau(t)}{\rho} \delta(z) * z \sum_{n=-\infty}^{n=+\infty} \delta(z-2 n h)$ \\
\hline $\begin{array}{l}\text { Solution } \\
\text { élémentaire }\end{array}$ & $Y(t) e^{-i f t}$ & $Y(t) e^{-i f t} e^{-\pi \frac{z^{2}}{4 \pi \nu t}}$ & $\sqrt{4}$ \\
\hline $\begin{array}{c}U \omega: \text { Réponse à la } \\
\text { fréquence angulaire } \\
\omega \neq-\dagger\end{array}$ & $\frac{\tau}{\rho} \quad \frac{1}{i(f+\omega)}$ & $\frac{\tau \omega}{\rho v} \frac{e^{-|z| \sqrt{\frac{i(t+\omega)}{v}}}}{\sqrt{\frac{i(f+\omega)}{v}}}$ & $\frac{\tau \omega}{\rho h} \quad \frac{a_{h}(\omega, z)}{i(f+\omega)}$ \\
\hline $\begin{array}{l}\text { Phase } \begin{aligned} \varepsilon & =+1 \\
\phi_{\omega}=\operatorname{Arg}(U \omega, \tau \omega) & =-1\end{aligned}\end{array}$ & $-\frac{\pi}{2}-\frac{\pi}{-\frac{\pi}{2}}$ & $\begin{array}{l}+\frac{\pi}{4}+|z| \sqrt{\frac{f+\omega}{2 v}} \\
-\left(\frac{\pi}{4}+|z| \sqrt{\frac{f+\omega}{2 v}}\right)\end{array}$ & $\arg \frac{a_{h}(\omega, z)}{i(f+\omega)}--\frac{a_{h}(\omega, z)}{i(f+\omega)}--$ \\
\hline $\begin{array}{ll}Y(t)= & \text { fonction d'Heavi } \\
\delta & \text { Distribution de } D \\
\tau & \text { Tension du Vent } \\
D_{\omega} & \text { Profondeur d'Ekm }\end{array}$ & $\begin{array}{l}\text { ide } \\
\text { rac } \\
\text { anec } E=+1\end{array}$ & $\begin{array}{r}D_{\omega}^{2}=2 \varepsilon \pi^{2} \\
\text { si } f+\omega>0 \text { si } \varepsilon^{+\infty}=-1 \text { si } \\
h(\omega, z)=\sum_{-\infty}^{+\infty} e^{\text {in } \pi / h}[\end{array}$ & $\begin{array}{l}v /(f+\omega) \\
\omega<0 \\
\left.-i \varepsilon n^{2}\left(\frac{D \omega}{h}\right)^{2}\right]^{-1}\end{array}$ \\
\hline
\end{tabular}
longueur $D_{\omega}$ est la profondeur d'Ekman généralisée, c'est-

Tableau I 




4/ Représentation graphique de $u_{\omega}$ pour $z=0$ et $z=-h$ en fonction de $h$ : l'unité de longueur est prise égale à $D_{\omega}$ et l'unité de temps à $T=2 \pi /|f+\omega|$. Quand $h$ devient très grand par rapport à $D_{\omega}$ on obtient́ la spirale d'Ekman, $U_{\omega}(z)$. La variation de $h$ ou de $z$ est matérialisée par les valeurs $m=32 h / D$ portées sur les 3 courbes $U_{\omega}(z=0), U_{\omega}(z=-h), U_{\omega}(z)$. Pour une couche de profondeur $m$ le rayon de l'arc de cercle $(m)$ matérialise l'amplitude de l'oscillation d'inertie correspondante. Pour $f+\omega<0$, les 3 courbes $U_{\omega}$ sont symétriques par rapport à $\tau_{\omega}$ de celles représentées ici (cas $f+\omega>0$ ).

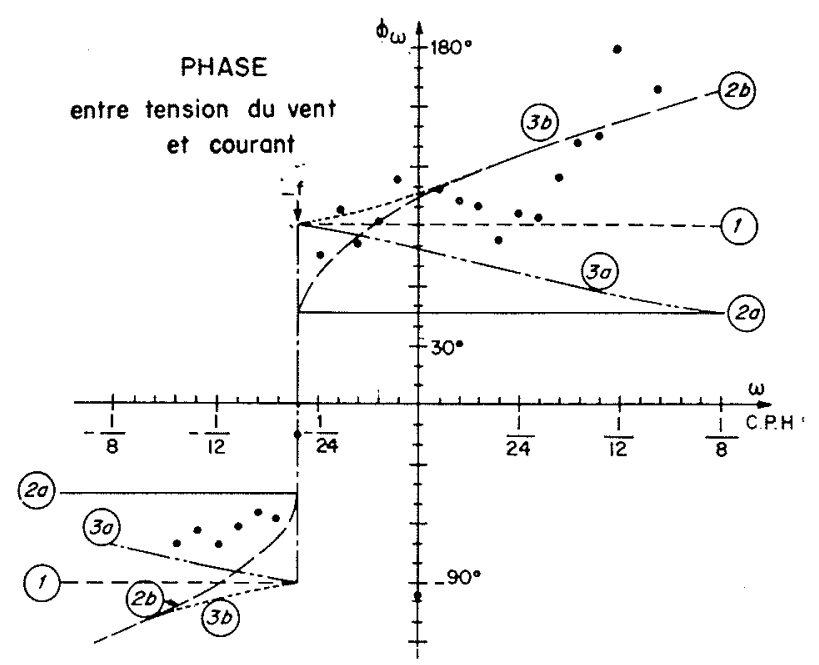
5/. Phaso $\phi_{\omega}$ ontra la tension dú vent ot lo courant dans la couche d'influenca : - In tigne (1) correspond au traneport to masso
- les lignes (2) sa rapportent à un océsn do profondeur infinia : ligna (20) pour is surfaca. ligne (2b) pour une profondour arbitrairo $z_{0}$ :
- las lignes (3) concernent un océan de profondeur finio $h=z_{0}$ : (3a) pour la surface. (3b) pour la profondour $h$
Las courbes (3a) et (3b) sont respectivement osymptstas a (2a) et (2b) quand $\omega \rightarrow \infty$. Las Points (.) sont donnés par l'analyso du vant ot du courant au site D. (ATLANTIQUE NH)
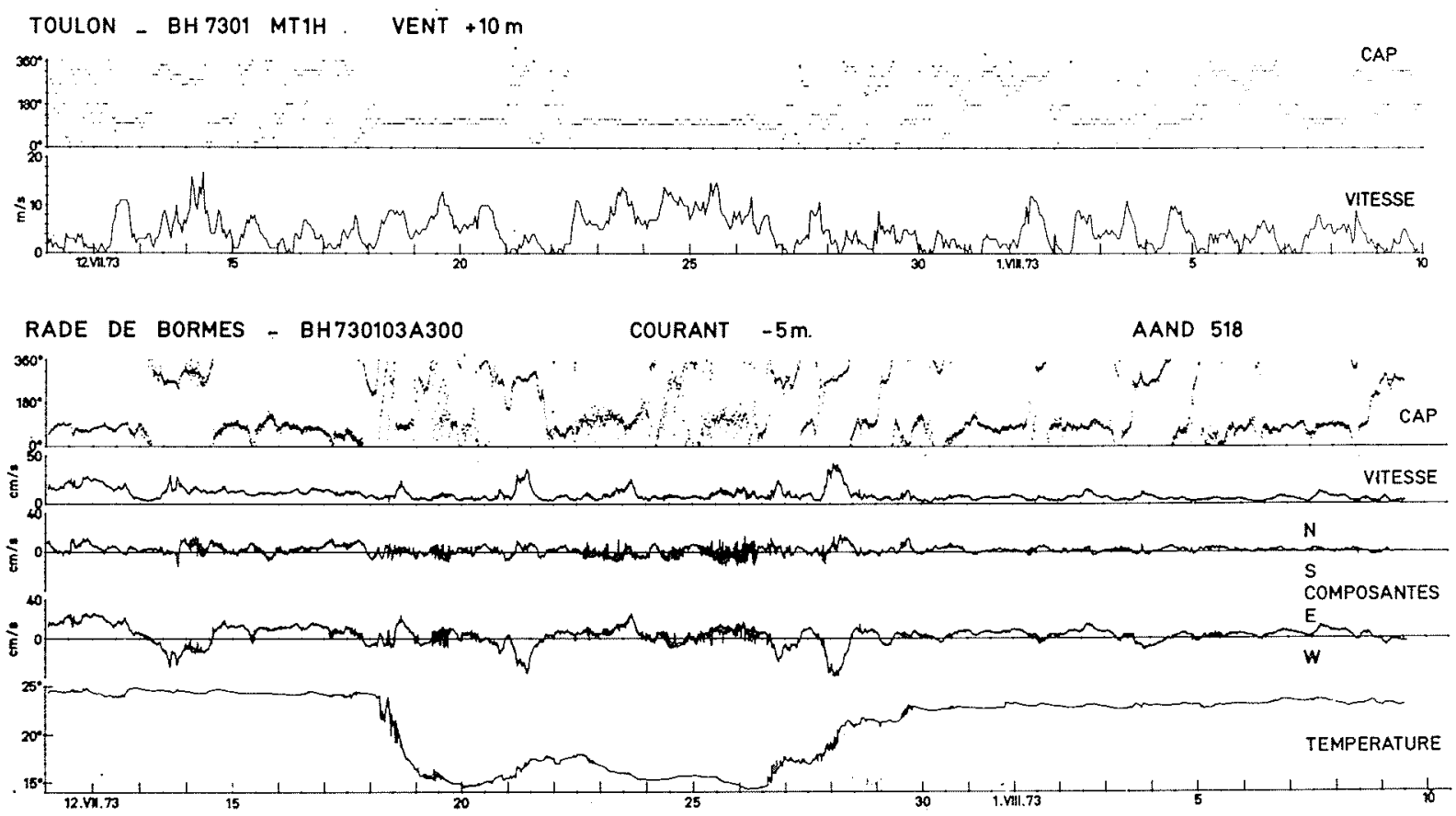
à-dire $D_{\omega}=D_{0}(f /|f+\omega|)^{1 / 2}$ où $D_{0}$ est la profondeur classique $\left(D_{0}^{2}=2 \pi^{2} \vee f\right)$. L'allure de la phase $\phi_{\omega}$ entre la tension $\tau_{\omega}$ et $u_{\omega}$ est donnée dans les différents cas par la figure 5 .

L'équation du mouvement suppose un océan illimité horizontalement et ne tient pas compte de la dissipation; la solution n'est représentative du phénomène que si l'action du vent est limitée dans le temps. Dans la nature, le vent n'est jamais permanent, il varie au cours du temps et se présente sous forme impulsive d'une durée $d$ limitée (ex. : le Mistral). L'amplitude de la composante $u_{--f}$ du courant (《oscillation d'inertie ») dépend des phases relatives des autres composantes $u_{w}$, mais la probabilité est grande pour que $u_{-f}$ soit la composante dominante et, en particulier, plus grande que la moyenne $u_{0}$ sur la durée $d$. Ceci explique pourquoi on observe dans la couche de mélange, donc en été, des «boucles» et non des ondulations dans l'hodographe intégré obtenu à partir des mesures de courant de surface (fig. 1 a et 3 , en haut).

Près des côtes, le phénomène est plus complexe. Les mesures de courant recueillies au cours de l'été 73 , à bord de la nouvelle bouée-laboratoire Borha $I I$, mouillée provisoirement en rade des Bormes par des fonds de $80 \mathrm{~m}$, sont représentées dans la figure 6 . Avec l'apparition du Mistral (le 18 juillet), on voit la température de l'eau qui tombe de 24 à $15^{\circ} \mathrm{C}$ en moins de 24 heures. L'examén détaillé de la composante E-W du courant (même direction que les isobathes) et la température, montre que la température décroît lorsque le courant porte à l'est et qu'elle croît lorsque le courant porte à l'ouest. Cette variation de température est associée aux variations du vent et on peut constater que les courants les plus forts sont ceux dus au retour à la côte des masses d'eau chaude lorsque le vent devient faible (21 juillet et 27-28 juillet). Ce phénomène d'upwelling associé au Mistral est assez analogue à celui observé au large des côtes de l'Orégon (R. L. Smith, 1974).

\section{Conclusion}

Les observations recueillies en Méditerranée à bord de la bouée-laboratoire ont montré que la théorie d'Ekman s'applique non pas à un océan homogène mais bien à un océan stratifié. Des doninées recueillies en mer du Nord ou en Atlantique Ouest donnent des résultats plus complexes du fait de la présence des marées. Mais l'apparition d'oscillations d'inertie est toujours liée au passage des perturbations atmosphériques (Webster; Pollard and Millard; Hunkins; Schott; Gonella).

En définitive, au large des côtes, il y a de fortes chances de trouver de bonnes corrélations vent-courant dans toutes les zones où l'on peut observer des oscillations d'inertie dans les couches superficielles. Ceci exclut les zones côtières, les étendues d'eau où l'homogénéité est atteinte sur de grandes épaisseurs et les zones proches des grands courants océaniques. Par contre, la corrélation vent-courant est plus nette quand l'apparition du vent se fait brutalement (ex. : coup de Mistral) et quand la couche de surface est fortement stratifiée. Le courant peut alors atteindire des amplitudes de l'ordre du mètre par seconde (courant moyeri + courant d'inertie).

Près des côtes, le phénomène est un peu plus complexe mais il semble, du moins en première approximation, que l'hypothèse de la couche d'eau chaude glissant sur la couche sous-jacente puisse être retenue pour l'étude des upwellings côtiers.

\section{Remerciements}

Ce travail a été réalisé dans le cadre d'un contrat de recherche avec le Centre National pour l'Exploitation des Océans. Les données météorologiques de Toulon ont été aimablement fournies par M. Romer, Ingénieur de la Météorologie Nationale.

\section{Bibliographie}

EKMAN. - Om jardrotationens inverkan pa vind-strömmer i hafvet (1902). Nyt. Mag. f. Naturvid, Vol. 40, Kristiania (1902).

GASCARD (J.-C.). - Vertical motions in a region of deep water (1973). Deep-Sea Res., 20, XI, pp. 1011-1028.

Gonella (J.). - Etude des courants marins de surface induits par le vent. Annales Hydrographiques, $5^{\circ}$ série, Vol. $1, \mathrm{n}^{\circ} 741$ (1973), fasc. 1.

Hunkins (K.). - Some inertial oscillations of Flctcher's Ice Island, T. 3 (1967). J. Geophys. Res., 72 (4), pp. 1165-1174.

Pollard (R.T.) and Millard $\mathrm{J}_{r}$ (R. C.). - Comparison between observed and simulated wind-generated inertial oscillations (1970). Deep-Sea Res., 17, pp. 813-821.

ScHotT $(\mathrm{F}$.$) . - On horizontal coherence and internal wave propa-$ gation in the North Sea (1971). Deep-Sea Res., 18 (3), pp. 291-308.

SMITH (R. L.). - A description of current, wind and sea level variations during coastal upwelling off the Oregon Coast, July-August 1972 (1974). J. of Geophys. Res., 79, 3, pp. 435443 .

Voorhis (A. D.) and WEBB (D. C.). - Large vertical currents observed in a winter sinking region of the North-Western Mediterranean (1970). Cah. Océanogr., XII, 6, pp. 571-580.

Webster (F.). - Observations of inertial period motions in the deep sea (1968). Rev, Geophys., 6 (4), pp. 473-490. 


\section{Discussion}

Président : M. J. DuporT

M. le Président remercie M. Gonella d'avoir bien voulu nous exposer ses intéressantes recherches théoriques et expérimentales et propose d'instaurer une brève discussion sur leurs différents aspects.

Sur une question de M. Gauthier, M. Gonella précise que, dans ses expériences, le courant était mesuré à $5 \mathrm{~m}$ de profondeur par fond de $80 \mathrm{~m}$, à 1 mille de la côte, au large du Lavandou. Il lui semble difficile de parler, ici, d'un phénomène «d'upwelling» permanent, en ce sens que «la plaque d'eau» superficielle, résultant de la stratification, est poussée au large par le mistral et remplacée temporairement par les eaux froides plus profondes.

Pour M. CRÉpon, il y a une liaison entre la remontée d'eau et la «plaque » susvisée. Il signale l'importance des phénomènes impulsionnels variables dans le temps mais, en général, non périodiques.

L'exposé de M. Gonella, observe M. le Professeur Lacombe, montre que la réponse au vent des couches superficielles marines dépend considérablement de la présence ou de l'absence d'une thermocline. L'exposé, que le même auteur nous fera cet aprèsmidi, de l'étude de M. GASCaRD nous permettra de mieux saisir cette différence de comportement.

M. Chabert-D'Hieres présente l'observation ci-après, qui ne se réfère pas spécialement à l'exposé de M. Gonelta, mais aussi à tous ceux qui l'ont précédé au cours de la séance :

Pour calculer la profondeur de la thermocline saisonnière M. le Professeur Lacombe a pris, dans le nombre de Richardson, le gradient de vitesse introduit par les mouvements orbitaux de houle. Ensuite, nous avons vu l'extrême importance des gradients verticaux de vitesse horizontale, engendrés par les ondes internes suivant les petits échelons de densité.

Quels sont les ordres de grandeur relatifs de l'un et de l'autre de ces paramètres? Quand les effets de la houle et des vagues l'emportent-ils sur les effets des ondes internes?

Je ne prétends pas, répond $M$. le Professeur LACOMBE, pouvoir défendre le choix de la houle parmi les autres facteurs. Comme je l'ai dit, c'est un peu intuitivement qu'il nous a semblé que le mélange pouvait être spécialement activé par la loule. Quels peuvent-être les effets des autres facteurs, tels que les ondes internes, pour provoquer ce mélange? La question reste tout à fait ouverte. Je ne voudrais pas que l'on considère le graphique que j'ai proposé, à la fin de mon exposé, comme un graphique définitif : c'est simplement une hypothèse qui paraît donner un ordre de grandeur qui n'est pas absurde.

Pourtant, observe M. COANTIC, on sait que les vitesses turbulentes sont à peu près proportionnelles à la vitesse de frottement. Si l'on considère qu'il y a conservation du frottement à l'interface, et si l'on tient compte du rapport des masses spécifiques, on s'apercoit que l'échelle des vitesses turbulentes dans l'eau est seulement $1 / 30^{\circ}$ de l'échelle des vitesses turbulentes dans l'air. Par ailleurs, on admet que les vitesses orbitales, induites dans l'atmosphère par les vagues, sont négligeables vis-à-vis de la turbulence; mais, dans l'Océan, en raison de l'importance de ce rapport - de 1 à 30 - il est tout à fait possible que les vitesses orbitales jouent un rôle privilégié vis-à-vis des fluctuations du courant.

Si la houle était parfaitement irrotationnelle et non turbulente, les vitesses orbitales ne devraient pas intervenir dans le nombre de Richardson. On observe cependant, en fait, un écoulement dans lequel les vitesses prépondérantes sont celles induites par la houle et, d'autre part, où cette houle est, par suite de la turbulence, légèrement non irrotationnelle. Je crois donc tout à fait fondé de baser un critère sur les vitesses orbitales.

M. J.-L. Suberville pose à tous les spécialistes de la thermocline la question - d'ordre général - ci-après :

Que pensez-vous de l'utilisation des modèles à deux couches superposées de densités constantes, généralement adoptés, pour décrire les phénomènes intervenant au niveau des thermoclines?

D’abord, répond $\mathrm{M}$. CRÉPoN, cette hypothèse est commode du point de vue mathématique... D'autre part, certaines mesures faites à bord de la bouée-laboratoire viennent confirmer cette hypothèse qui semblait, a priori, un peu simpliste.

Cela dépend des lieux et des époques, précise M. SaInt-Guily. La représentation par un modèle à deux couches est bonne quand il y a une forte thermocline bien marquée, en été par exemple, en Méditerranée. Par contre, elle est beaucoup moins bonne en hiver.

M. CRÉPON et M. SAINT-Guily sont d'accord pour estimer que l'on pourrait tenter un meilleure approche des phénomènes en supposant une densité constante au-dessus de la thermocline et une densité décroissante - suivant une exponentielle? - au-dessous.

M. J.-L. Suberville signale que ses travaux personnels l'ont conduit à étudier un modèle théorique plus évolué, qui lui semble proche des conditions naturelles, comme le prouvent certains profils verticaux relevés en mer. Le modèle proposé ne prétend, en aucune façon, résoudre tous les problèmes qui se posent, mais présente l'avantage de prendre en compte un paramètre supplémentaire, qui est celui de la croissance de la masse volumique avec la profondeur, au-dessous de la thermocline (la masse volumique de la couche supérieure restant constante). Cette croissance est supposée exponentielle, ce qui permet un traitement mathématique relativement simple (fréquence de Brunt-Väisälä constante).

En raison des impératifs de l'horaire, $M$. le Présiclent clôt la discussion et la séance en remerciant vivement les conférenciers ainsi que les personnes qui sont intervenues dans la discussion pour la qualité et l'intérêt de leurs exposés.

La séance est levée à 12 h 15 . 